\title{
Effect of pressure on the physical properties of magnetorheological fluids
}

\author{
A. Spaggiari, E. Dragoni \\ Dept. of Engineering Sciences and Methods, University of Modena and Reggio Emilia, Italy \\ andrea.spaggiari@unimore.it
}

\begin{abstract}
To date, several applications of magnetorheological (MR) fluids are present in the industrial world, nonetheless system requirements often needs better material properties. In technical literature a previous work shows that MR fluids exhibit a pressure dependency called squeeze strengthen effect. Since a lot of MR fluid based devices are rotary devices, this paper investigates the behaviour of MR fluids under pressure when a rotation is applied to shear the fluid. The system is designed in order to apply both the magnetic field and the pressure and follows a Design of Experiment approach. The experimental apparatus comprises a cylinder in which a piston is used both to apply the pressure and to shear the fluid. The magnetic circuit is designed to provide a nearly constant induction field in the MR fluid. The experimental apparatus measures the torque as a function of the variables considered and the yield shear stress is computed. The analysis of the results shows that there is a positive interaction between magnetic field and pressure, which enhances the MR fluid performances more than twice.
\end{abstract}

KEYWORDS. Magnetorheological fluids; Shear mode; Pressure; Design of experiment.

\section{INTRODUCTION}

$\mathrm{M}$ agnetorheological (MR) fluids are smart materials that are increasingly used in many applications, such as controllable clutches and dampers [1]. An external magnetic induction field reversibly changes the apparent viscosity of MR fluids. MR fluids are capable to switch from a free-flow liquid at no induction, to a quasi solid state when a strong magnetization is present. The quickness of change (5-10 milliseconds) when a magnetic field is applied, makes this material interesting for adaptive damping and dissipative applications.

MR fluids can be used to build silent, fast and tunable mechanical devices, which are enhanced by the ease of integration with the electronic control unit. The MR effect is obtained by a 30-40\% in volume of ferromagnetic particles dispersed in the carrier fluid (silicon or hydrocarbon oil). When a magnetic field is applied to MR fluids, the particles are subjected to a dipole magnetic moment and align with the flux lines. Consequently there is a formation of linear chains of particles (at a micro-scale) which means that, at the macro-scale, the MR fluid becomes a solid-like material.

One of the most important design parameter of MR fluids is the yield shear stress $\left(\tau_{y}\right)$, which is the maximum stress the fluid can withstand before shear occurs. This value is fundamental in the design of any MR fluid devices, because the higher is the stress the higher is the dissipated power of the system. Since many MRF devices are dissipative (e.g. dampers) the higher the power the better the performance.

The yield shear stress, $\tau_{y}$ is controlled by the magnetic field, as shown in the technical datasheet supplied by the producer (Lord Corporation [2]) for the commercial MR fluid 140-CG. There is a magnetic saturation of the magnetic particles in MR fluids at high magnetic flux levels [2] which causes a typical sigmoid shape of the B-H curve of the material as reported in the producers datasheets. This saturation leads to a limitation of $\tau_{y}$, and unfortunately the dissipated power reaches a maximum no matter how high the magnetic field is. It is possible to increase the value of $\tau$ y by changing the 
volume fraction of the particle in suspension, but also in this case there is a physical limit, otherwise the viscosity of MR fluid would increase too much.

A finite element analysis was performed by Ginder et al. [3] to study the effect of magnetic nonlinearity and saturation of magnetic particles. They found a limit for the volume fraction at $50 \%$ in volume, which gives a maximum of $\tau_{y}=210 \mathrm{kPa}$. In any case for industrial applications, a value of 50\% volume fraction is too high, and the normal volume fraction ranges from $20 \%$ to $40 \%$, which corresponds to a typical iron particle content in weight of $75 \%-85 \%$ ) as reported in Lord Corp. datasheets [4-5]. MR fluids in squeeze mode (with the magnetic field acting in the same direction of the movement) provide higher forces compared to shear and flow mode. The main drawback is that squeeze mode enables only very short strokes to be performed and this prevents MR fluid application in many industrial contexts.

A MR fluid compressed along the direction of the magnetic field, according to Tang et al [6], shows an increment of $\tau_{y}$ of nearly ten times. The explanation is the formation of thicker and thus stronger columns of particles that are able to sustain the load. Zhang et al. [7] designed an apparatus to evaluate the effect of a mechanical compression on MR fluids in linear shear mode. The apparatus they used, described in [7], which was quite complex and bulky, consisted of a non magnetic container for the MR fluid compressed through a big bolt, and a metal sheet was pulled off from the container to assess the shear strength under several pressure levels. Their experimental campaign revealed that a very high compression could enhance $\tau_{y}$ by more than 20 times for a given magnetic field, thus showing a so called squeeze strengthen effect. They also correlated this behaviour not only with the magnetic force of the dipoles formed by the ferromagnetic particles, but also with the friction between the particles. A hybrid tribological-magnetic model provided a good explanation of the very high yield stress data retrieved in the experiments. The authors previous work on the squeeze strengthen effect in flow mode [17] highlights the beneficial effect of the pressure on the MR yield stress.

The aim of this paper is to estimate the squeeze strengthen effect of an MR fluid under a shear stress, obtained by a relative rotation of the parts. The majority of the MR fluid based device working in shear mode are rotary devices like brakes and clutches and their performances would improve by exploiting the squeeze strengthen effect. Hence, a rotary experimental test is needed to gather experimental data on the influence of magnetic field and pressure, possibly using and architecture close to the common industrial application. Moreover this architecture gives a useful insight on important industrial aspects, like chemical compatibility of the sealing and geometrical tolerances needed to hold the MR fluid under pressure.

The behaviour of Lord 140-CG [2] commercial fluid was investigated under several magnetic field and pressure values using an ad-hoc apparatus. A design of experiment technique [8] was applied to the experimental tests in order to verify statistically the influence of the variables and to provide an empirical relationship to link the pressure, the applied field, and the yield shear stress.

A surface response was obtained on the basis of the experimental points and a design equation which correlates yield stress, pressure and induction field was provided.

The experimental results cover magnetic induction up to $300 \mathrm{mT}$ and pressure levels up to 30 Bar. The internal pressure influences the yield stress and there is a positive interaction between the magnetic field and the pressure. This study confirms that the findings of [7] are also valid under a rotational shear stress and thus there is the chance to increase MR fluid based clutches and brakes by simply changing the working pressure of the fluid.

\section{METHOD}

\section{Experimental set-up}

7 his section outlines the design of the experimental apparatus and describes the architecture of the system implemented to test the MR fluids. The cross-section of the magneto-hydraulic system used in the experiments is depicted in Fig. 1. The system consists of a lower frame (7), which is welded to the bottom flange (8) used to couple with the load cell of the testing machine. Another flange (6) is welded to the frame and is used to support the external tube (3), which is made in ferromagnetic material and is used to close the path of the magnetic flux (See Electromagnetic system section), as well as the upper flange (2). The MR fluid vessel (10), which is supported by the lower frame, has three main functions. First it contains the MR fluid, second it is used to enforce the coupling with the pressure transducer (9) and third it allows the correct alignment with the central piston (1). The pressure sensor (9) is a KellerDruck 25Y piezoresistive flush transducer [11], which exhibits a particular architecture compatible with MR fluids. Thanks to its stainless steel flat membrane, used as a sensitive element, the sensor is not affected by the presence of the microparticles and moreover its $\mathrm{G}^{1} / 2$ threaded connection ensures the correct sealing of the fluid. The upper sealing system (5) is made by a Polypac ring (Fig. 1 in black) [10], which is a commercial system for sealing liquids under pressure. 
The Polypac ring consists of an external O-Ring and an internal PTFE ring reinforced with glass fibres. The O-Ring is energized by the fluid and hence its compression inside the groove pushes the PTFE ring on the piston (1). The ring material provides both the sealing and low friction. This system allows the central piston to be moved up and down to generate the desired pressure and to rotate in order to shear the MR fluid. The torque measure will be affected by the different friction due to the pressure sensitive sealings (due to different pressure levels) and proper techniques will be used to extrapolate the correct yield shear stress.

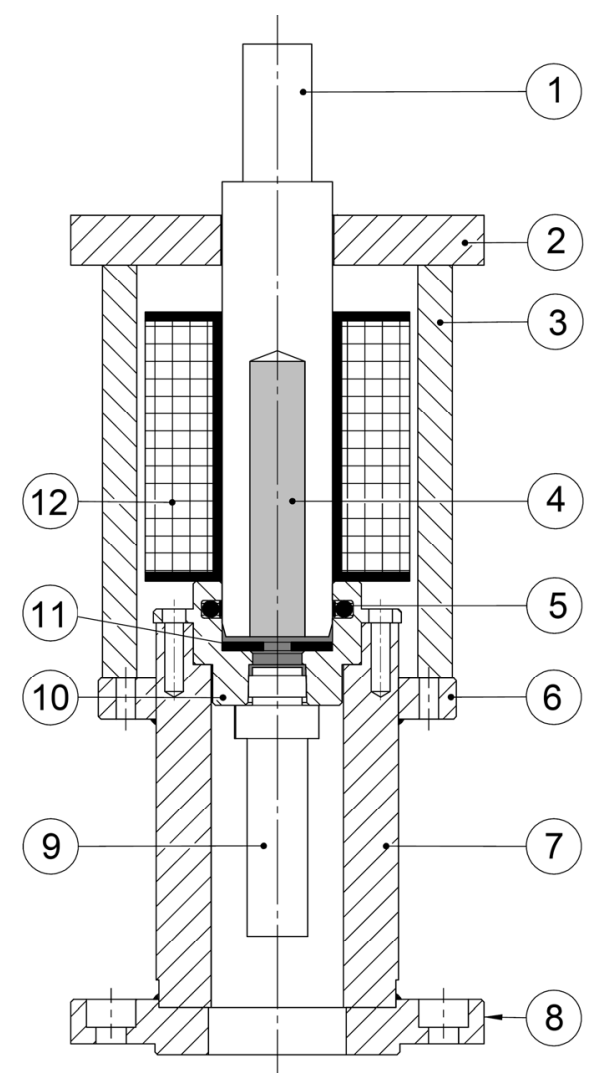

Figure 1: Cross-section of the hydraulic system.

The central piston (1), shown in Fig. 1 (not cross-hatched), has a deep central hole which is used to focus the magnetic field only into an annular area in conjunction with the washer (11) bonded to bottom of the vessel (10). Parts (1) and (11) are made in low carbon steel, with high magnetic permeability, while the vessel (10) is made in brass, amagnetic, likewise the central plug (4) made in PTFE, represented in light grey in Fig. 1. The PTFE is useful to prevent accidental torque transmission between the central part of the piston where the magnetic field is low and no torque should be present. The MR-fluid 140-CG by LORD corporation [2] is entrapped between (10), (9), (5) and is shown in dark grey in Fig. 1. The magnetic field is applied with the coil (12) which consists of 1932 turns made in AWG22 wire, wounded around a plastic housing (in black). Further details about the magnetic properties of the system, the flux path, and the applied currents are provided in the Electromagnetic system section. The small chamfer of the central piston (1) is necessary to prevent damage of the PTFE ring during the insertion of the piston through the Polypac (5) seal [10]. The system is designed to have a MR fluid chamber with height of $1 \mathrm{~mm}$ at the maximum pressure. The MR fluid used [2] is based on silicon oil which is more compressible compared to other hydrocarbon oils. This peculiarity makes the system easier to control because the pressure is changed by moving the central piston, and the little compliance given by the fluid prevents dangerous overloading.

The wires coming out from the coil exit from the central part of the system through holes in element (2), not visible in the cross section in Fig. 1, in order to be connected to a DC power source.

The experimental apparatus provides a rotational shear mode test of MR fluids under several levels of internal pressure. The design of the system is completely different Zhang's [7], and is much more compact and easy to manufacture. The electro-magnetic coil is displaced around the central ferromagnetic piston and is used to vary the magnetic excitation in the fluid (see Section Shear stress in the MR fluid). The system was placed under a universal biaxial machine, MTS Bionix 
858, which is able to compress the fluid applying the desired pressure and to shear the fluid through a rotation at the same time. The tensile machine is controlled both in compression, to hold the same pressure during the test and in angle, meanwhile the torque is measured.

From the recorded data, the yield stress values were calculated with analytical formulas and the effect of the pressure was estimated. The tests were performed at low rotational speed to limit viscous effects and to focus exclusively on the yield shear stress. In magnetorheological applications (e.g. dampers) the magnetorheological effect is from ten to hundrends times higher than viscous one. Therefore for the purpouse of this quasi static analysis the viscous effect are neglected. The shear rate, the pressure values applied and the magnetic field values are described in the subsequent sections.

The experimental test equipments are displayed in Fig. 2. Fig. 2a shows the complete system mounted on the MTS Bionix 858, with the TTi DC current supply on the left and the red display to monitor the pressure inside the MR fluid chamber in the centre. The MR fluid is placed inside the brass vessel and then, using the upper grip of the tensile machine the central piston is applied to seal the circuit (see Fig. 2b). The fluid volume is constant, the thickness is slightly changed to achieve the desired pressure level, but this small change do not affect the magnetorheological behaviour of the system.

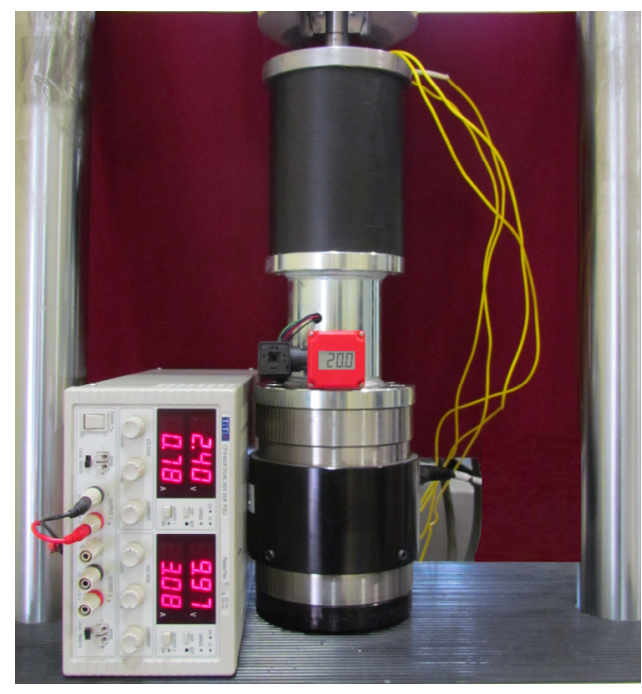

(a)

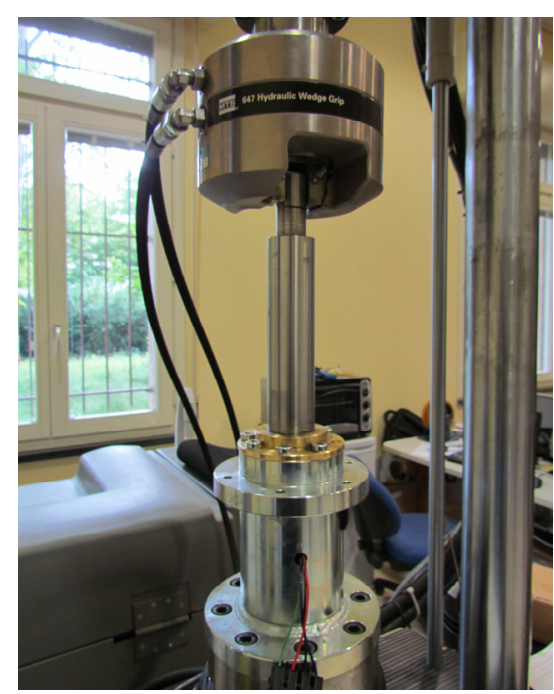

(b)

Figure 2: MR fluid insertion (a) central ferromagnetic piston fit (b) and full system mounted on the tensile machine.

\section{Shear stress in the MR fluid}

MR fluids, especially for quasi-static applications, can be conveniently modeled as Bingham fluids [9]. The typical behaviour of such a fluid is described by the solid line in Fig. 3 in terms of shear stress versus shear rate. The fluid exhibits a yield stress $\tau_{y}$ at no shear rate, and only when this value is reached the MR fluid starts to flow like a classic Newtonian fluid. The considered model involves two parameters: $\tau_{y}$ and the fluid viscosity $\eta$. The Bingham model shows the basic function of the MR fluid, but does not take into account shear thinning or thickening whereas other more complex model like the Herschel-Bulkley one does [12]. The $\tau_{y}$ value is a function of the applied magnetic field, B. Since the geometry of the chamber containing the MR fluid is quite simple the shear stress calculation can be done through analytical considerations.

$$
\tau=\tau_{y}(B)+\eta \dot{\gamma}
$$

The angular rotation is applied with a very low speed, $5^{\circ} / \mathrm{min}$, with a corresponding shear rate under $1 \mathrm{~s}^{-1}$, so the procedure can be considered quasi static. This makes the pure viscous effects negligible.

The MR fluid is active only where the magnetic field is on and the particles are aligned with the flux lines. The magnetic system is designed to focus the field only in an annular area with internal radius $R_{\text {int }}=10 \mathrm{~mm}$ and external radius $R_{\text {ext }}=20 \mathrm{~mm}$. The yield shear stress is considered constant because the magnetic field is constant, as shown in the magnetic electromagnetic simulation section, and acts on the annular area, $A_{f}$ which is:

$$
A_{f}=\pi \cdot\left(R_{\text {ext }}^{2}-R_{\mathrm{int}}^{2}\right)
$$




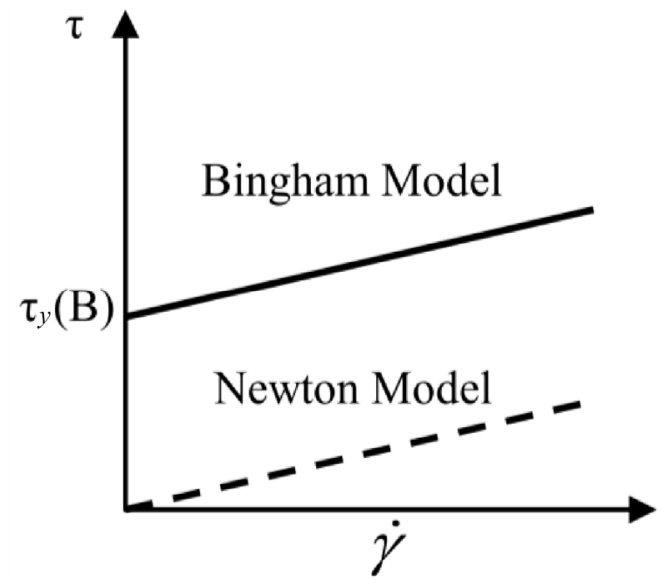

Figure 3: Bingham model, suitable for MR fluids and the classical viscous Newton model.

The static torsional moment due to the yield shear stress acting on the frontal annular area is:

$$
T=\int_{R_{\text {int }}}^{R_{\text {ext }}} \int_{0}^{2 \pi} \tau_{y} r^{2} d r d \theta=2 \pi \tau_{y} \frac{R_{e x t}^{3}-R_{\text {int }}^{3}}{3}
$$

The chamfer present in the central rod creates another active lateral cylindrical surface on which the yield stress $\tau_{y}$ is acting. The axial length of the chamfer is $L=2 \mathrm{~mm}$ and the cylindrical lateral area, $A_{l}$ is thus:

$$
A_{l}=2 \pi R_{\text {ext }} L
$$

According to the magneto-static analyses shown in the subsequent section the magnetic field in the frontal area is similar to the one in the lateral area.

Hence the shear stress can be computed considering the effect of the applied torque, $T$, over the two above calculated surfaces:

$$
\tau_{y}=\frac{T}{A_{l} \cdot R_{e x t}+2 \pi \frac{R_{e x t}^{3}-R_{\text {int }}^{3}}{3}}
$$

The pressure is regulated using the central piston until the desired value is reached. After that the system control keeps the piston still in the axial direction and the pressure is maintained constant. Then the central piston rotates and the fluid is sheared. The measured torque is caused both by the yield stress of the fluid according to Eq. (5) but also by the friction of the sealing system, which is pressure dependent.

The friction due to sealing is quite difficult to consider analytically and consequently it will be handled by considering a particular experimental procedure useful to eliminate any effect not related to the magnetic field and the internal pressure. The experimental procedure is organized in five steps:

1. The current is turned on at the beginning of the test to create the magnetic field.

2. The machine applies a prescribed rotation up tp to $2.5^{\circ}-3.5^{\circ}$ and records the total (gross) torque.

3. The current is suddenly turned off, so the torque values drops down because the MR effect has vanished.

4. The torque due to pure friction is measured.

5. The net torque is computed by subtraction of the two values of torque measured in step 2 and 4.

This procedure allows the pure MR fluid shear stress to be calculated disregarding all the frictional effects since the only difference between the first and the second part of the test is due to the current and thus to the MR effect.

Electromagnetic system

Applying a magnetic field correctly is a key point in exploiting the potential of any kind of MR fluid. The main problem in magnetic circuit design is to avoid flux losses due to flux dispersion in non ferromagnetic material (e.g. air). Since the application is quasi static there are no other losses due to eddy currents The first problem in dealing with MR fluids is that the fluid itself has low magnetic properties. The relative magnetic permeability can be retrieved by the producer technical specification [2]. According to electromagnetism fundamentals, the induction field $B$ is a function of the magnetic field $H$ and the magnetic permeability, as shown in Eq. (6) 


$$
B=\mu_{0} \cdot \mu_{r} \cdot H
$$

Hence, by approximating the $B-H$ curve linearly (assumption valid when $H<200 \mathrm{k} A / \mathrm{m}$ ) the value of the relative magnetic permeability is:

$$
\mu_{r}=\frac{B}{\mu_{0} \cdot H}=\frac{B}{4 \pi \cdot 10^{-7} \cdot H}
$$

As can be seen in the producers datasheets [4] when $H=125000 \mathrm{~A} / \mathrm{m}, B=0.8 \mathrm{~T}$ the relative permeability is $\mu_{r}=5.09$. The comparison between the linear approximation and the non-linear curve is not reported for the sake of brevity, however a linear approximation with $\mu_{r}=5$ is in good agreement $\left(R^{2}=0.978\right)$ with the manufacturer's curves [2] in the hypothesis that $\mathrm{H}<200 \mathrm{kA} / \mathrm{m}$. The value of $\mu_{r}=5$ is considered in the analysis, which is also in accordance with the literature [12][13].

The magnetic system was designed using finite element magnetic software, FEMM 4.0 [14], which is useful for two dimensional magnetostatic problems. Since the software is $2 \mathrm{D}$ only, the analyses were run in axisymmetric mode, in order to consider the complete geometry. The material choice is crucial in designing magnetic circuits. The main parts (1-2-3-67-8) were realized in low carbon steel, because of its high magnetic permeability. The only non magnetic part in addition to the copper coil is the brass vessel (10). The first FEMM analyses, not reported for the sake of brevity, showed that using a ferromagnetic vessel would have deviated the magnetic flux from the MR fluid. Thus the use of amagnetic materials in the system design was needed to force the flux to go inside the MR fluid.

The brass was chosen because it is a good tradeoff between mechanical strength, cost and amagnetic properties. In order to complete the magnetic analyses only the coil properties are needed. Since the copper wires were AWG 22 (wire diameter of $0.64 \mathrm{~mm}$ ) there current density was limited only by thermal considerations, not critical for this particular applications.

\section{Design of experiment}

Applying a statistical method can be convenient in dealing with experimental problems involving multiple variables. The Design of Experiment procedure, a well-founded statistical method based on the analysis of variance (ANOVA) [15], can be applied to such scientific problems as shown in [16]. In this experiment the variables involved are the applied magnetic induction field $(B)$ and the internal pressure of the MR fluid $(p)$.

Literature results showed an interaction between the two variables both in linear shear mode [7] and in flow mode [17]. The experiments were designed to verify the same interaction under a rotational loading, which is the typical configuration of brakes and clutches. The most frequent application of the Design of Experiment is the two-level factorial experiment, which is used mainly for exploratory experiments in order to obtain quick qualitative insights on the process. In this work, on the other hand, we adopted the "general factorial" approach, which allows to consider multiple levels per variables. In this case four levels are considered for both pressure and magnetic field, and it is possible to capture eventual non linearity of the variables. The method is focused only on two variables which influence the behaviour of the system, it is able to identify the interaction between these variables more precisely, and is able to provide a more reliable model to describe how the system behaves. The magnetic induction ranges from 0 to $300 \mathrm{~m}$ T, while the internal pressure spans the $0-30 \mathrm{Bar}$ range.

The complete experimental plan is reported in Tab. 1. The experimental tests consider explicitly only non zero value of magnetic induction field, mainly because of the particular differential procedure adopted to calculate the net torque as described in the previous section. The zero level is intrinsically obtained in each test when the current is turned off according to the above described procedure. The experiments at zero current give only information on the pure frictional forces since the viscous effect is negligible due to quasi static rotation of the central piston (1).

\section{Electromagnetic simulation results}

The simulation of the magnetic system using FEMM 4.0 at a current of $2.3 \mathrm{~A}$ is reported in Fig. 4a. The flux lines passes through the MR fluid as requested and the magnetic field, thanks to the presence of the PTFE central plug (4) assumes an annular shape as desired.

The system provides a magnetic induction field in the MR fluid up to $300 \mathrm{mT}$ with a supply current of 2.3 A. The distribution of the magnetic induction field inside the MR fluid is provided in Fig. 4b, confirming the nearly constant value in the annular region and a lower value in the central part of the system. The electromagnetic simulation, like the one showed in Fig. 4a, were carried out for the four values of magnetic induction field considered. 


\begin{tabular}{ccccc}
\hline Levels & I & II & III & IV \\
Induction Field, $B,(\mathrm{mT})$ & 50 & 100 & 200 & 300 \\
Internal Pressure, $p$ (Bar) & 0 & 10 & 20 & 30 \\
Replicates & 3 for each combination \\
Experimental Points & \multicolumn{3}{c}{16} \\
Grand Total & 48 \\
\hline
\end{tabular}

Table 1: Experimental plan.
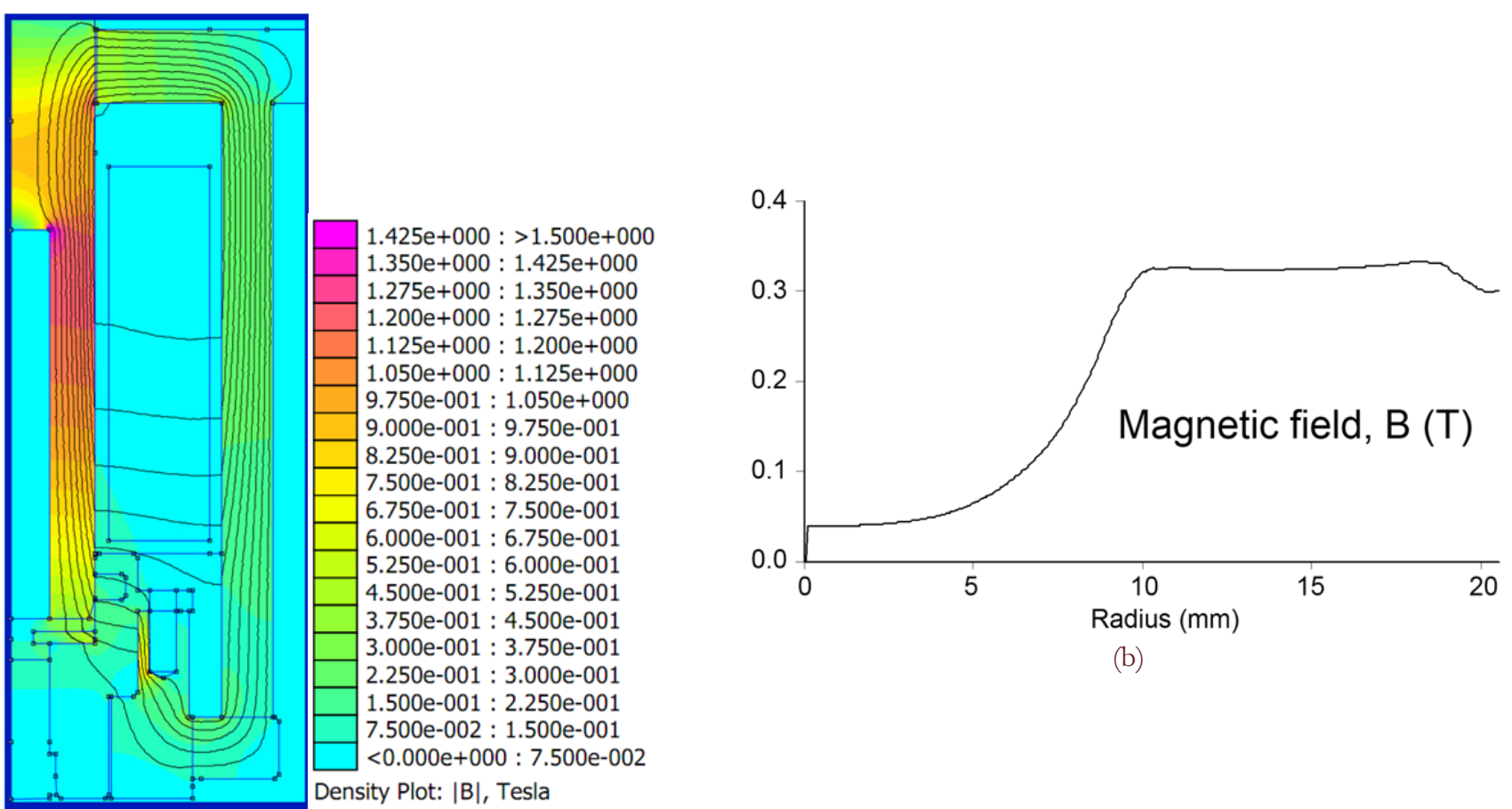

(b)

(a)

Figure 4: Flux density inside the hydro-magnetic system for a current of 2.31 Amp (a), scale 0-1.5T, and flux distribution along the radius of the MR fluid chamber (b).

The direct measurement of the magnetic field inside the MR fluid is not possible for two reasons. There is no way to access the MR fluid chamber when the system is completely assembled because it is perfectly sealed. Even if it was possible the hall effect probe of the Gaussmeter Hirst GM05 would not have been compatible with MR fluid, since the micronsized particles are almost impossible to be cleaned and the subsequent magnetic measure would have been affected by their presence.

In order to verify the FEMM predictions of Fig. 4 two indirect experimental measurements were done on the system. The first measures were done without the fluid, removing the pressure transducer (9) an accessing the chamber from the bottom threaded hole with the flexible Hall effect probe of the Gaussmeter.

The second measures were performed on the complete system putting the thin Gaussmeter probe in the gap between part (1) and (2) and comparing the FEMM values with the experimental ones.

\begin{tabular}{ccccccc}
\hline Current (A) & \multicolumn{3}{c}{ First point, inside } & 10), no MRF & \multicolumn{3}{c}{ Second point, in the gap between (1) and (2) } \\
& Exp. & FEMM & Difference & Exp. & FEMM & Difference \\
0.385 & 24.7 & 26.275 & $6.38 \%$ & 54.84 & 57.375 & $4.62 \%$ \\
0.77 & 52.5 & 52.55 & $0.10 \%$ & 105.2 & 114.75 & $9.08 \%$ \\
1.54 & 105.1 & 107.98 & $2.74 \%$ & 212.57 & 229.5 & $7.96 \%$ \\
2.31 & 210.2 & 217.74 & $3.59 \%$ & 426.5 & 459 & $7.62 \%$ \\
\hline
\end{tabular}

Table 2: Experimental and FEMM values of the induction magnetic field (mT). 
The collected and the simulated values are reported in Tab. 2. The FEMM model is able to provide a good prediction of the magnetic field both in the first access point and in the second. The numerical prediction is always larger than the experimental values, probably due to an overestimate of the magnetic permeability of the steel used to manufacture the experimental test fixture.

\section{RESULTS AND DISCUSSION}

\section{Experimental results}

I

$\mathrm{n}$ order to explain the post processing of the raw experimental data a typical torque-angle raw curve is reported in Fig. 5. As highlighted in the red circle the torque suddenly drops down in the middle of the test. This is because the current is turned off in order to obtain two field levels from the same test. The net torque is obtained upon subtraction of the average torque level in the second part of the diagram from the first one. The net torque is significantly lower than the absolute torque, this behaviour is mainly due to sealing chosen in the experimental system. Since it was much important to prevent the leakage of the fluid with several pressure values, rather than having low frictional forces, the seal provided an extraforce which could be subtracted a posteriori.

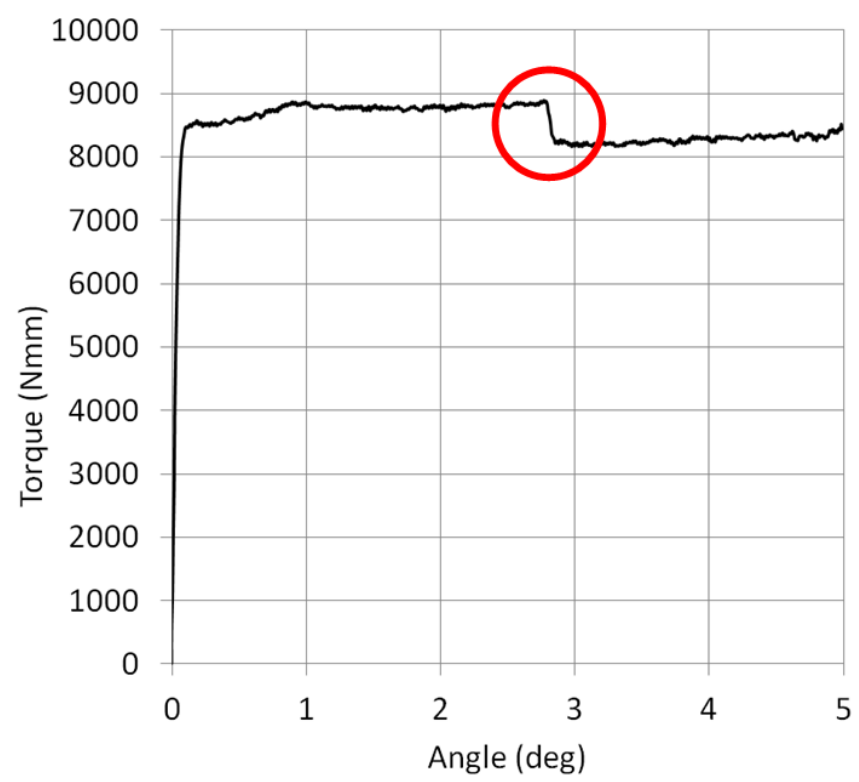

Figure 5: Typical raw experimental torque-angle curve. The red circle highlights the moment of the current turn off.

The procedure is applied for all the 48 experimental test curves obtained from the design plan and the results in terms of net torque are reported in Fig. 6. Fig. 6a shows the curves obtained at ambient pressure, Fig. 6b-c-d shows the curves at 10-20-30 Bar respectively.

This procedure allows retrieving only the difference in torque levels, while the difference of in the turning off angle (reported along the x-axis), visible in Fig. 6 is not an issue. The complete set of experimental torque values are reported in Tab. 3, as well as the average net torque value and the average shear stress valued obtained from application of Eq. (5). The experimental results shows some high dispersion of the data, but, on the whole the standard deviation is around $12 \%$ of scatter. Two possible explanation are envisioned. First some stick slip effect on the sealing could have occurred despite the Teflon ring used to ensure the seal, second a residual magnetization of the particles could have influenced some outlier experimental points (e.g. $p=10$ Bar, $B=100 \mathrm{mT}, \mathrm{R} 1$ ). This dispersion seems acceptable since the experimental intrinsic scatter do not change the general trend of the system under magnetic field and pressure.

The main difference between the net torque curves of Fig. 6 and the raw data of Fig. 5 is that the there is an important offset which is disregarded in the shear stress measure. The post processing procedure is justified by the fact that $\tau y$ do not depend on the frictional forces, but particular attention must be paid to the fact that the higher the pressure, the higher the forces due to sealing. 


\begin{tabular}{|c|c|c|c|c|c|c|c|c|}
\hline \multirow[b]{2}{*}{$\begin{array}{c}\mathrm{P} \\
\text { (Bar) }\end{array}$} & \multirow[b]{2}{*}{$\begin{array}{c}\mathrm{B} \\
\left(\mathrm{m}^{\prime} \mathrm{T}\right)\end{array}$} & \multicolumn{5}{|c|}{ Net Torque (Nmm) } & \multicolumn{2}{|c|}{$\tau_{y}(\mathrm{kPa})$} \\
\hline & & $\mathrm{R} 1$ & $\mathrm{R} 2$ & R3 & Average & Std. Dev. & Average & Std. Dev. \\
\hline 0 & 50 & 172.08 & 213.56 & 146.21 & 177.28 & 33.97516 & 9.25 & $19.16 \%$ \\
\hline 0 & 100 & 514.47 & 543.45 & 507.13 & 521.68 & 19.20442 & 27.22 & $3.68 \%$ \\
\hline 0 & 200 & 1204.06 & 998.46 & 1168.5 & 1123.67 & 109.8859 & 58.64 & $9.78 \%$ \\
\hline 0 & 300 & 1156.95 & 1020.73 & 1393.3 & 1190.33 & 188.5142 & 62.12 & $15.84 \%$ \\
\hline 10 & 50 & 255.78 & 225.71 & 248.84 & 243.44 & 15.74466 & 12.70 & $6.47 \%$ \\
\hline 10 & 100 & 915.54 & 482.43 & 445.15 & 614.38 & 261.4832 & 32.06 & $42.56 \%$ \\
\hline 10 & 200 & 1658.38 & 1704.4 & 2001.82 & 1788.20 & 186.4258 & 93.31 & $10.43 \%$ \\
\hline 10 & 300 & 2374.80 & 2683.51 & 2688.13 & 2582.15 & 179.5823 & 134.75 & $6.95 \%$ \\
\hline 20 & 50 & 198.97 & 229.18 & 157.53 & 195.23 & 35.97138 & 10.19 & $18.43 \%$ \\
\hline 20 & 100 & 605.44 & 665.52 & 573.83 & 614.93 & 46.57584 & 32.09 & $7.57 \%$ \\
\hline 20 & 200 & 1901.04 & 2037.91 & 2084.71 & 2007.89 & 95.44484 & 104.78 & $4.75 \%$ \\
\hline 20 & 300 & 2824.34 & 2523.62 & 2949.16 & 2765.70 & 218.7452 & 144.32 & $7.91 \%$ \\
\hline 30 & 50 & 237.58 & 249.54 & 291.72 & 259.61 & 28.44097 & 13.55 & $10.96 \%$ \\
\hline 30 & 100 & 931.46 & 753.29 & 739.61 & 808.12 & 107.0344 & 42.17 & $13.24 \%$ \\
\hline 30 & 200 & 2347.10 & 1808.65 & 2181.02 & 2112.25 & 275.7325 & 110.22 & $13.05 \%$ \\
\hline 30 & 300 & 2992.63 & 3084.06 & 2802.75 & 2959.81 & 143.4975 & 154.45 & $4.85 \%$ \\
\hline
\end{tabular}

Table 3: Experimental net torque and yield shear stresses $\tau_{y}$ retrieved using Eq. (4).

Both from the experimental curves in Fig. 6 and the experimental data in Tab. 3 it is possible to qualitatively sense that the pressure does have an important effect on the shear stress and it is a positive effect. Since a design of experiment procedure was applied the data are analyzed applying an Analysis of Variance: this statistical method is able to quantify variable influence and variable interaction on the process under scrutiny.

\section{Analysis Of Variance On The Yield Stress}

An analysis of the variance was applied using Design Expert 8.0 software [18]. ANOVA calculates the variance of a response by considering a specific variable and the global variance in the responses. Among the possible approaches to graphically represent the results, one of the most popular is the normal plot, which is used to estimate whether a certain set of data follows a Gaussian distribution or not. If the data approximates a straight line, the phenomenon is statistically "normal" i.e. follows a stochastic law and can be attributed to background noise. The variables or the interactions affecting the system's behaviour will then fall outside the normal distribution line, thus their effect cannot be ascribed to a stochastic process. The greater the deviation of the point from the normal line, the larger the confidence interval (i.e. the probability that the variables are significant). The half normal plot used in this paper is interpreted in the same way as the normal plot, but allows absolute values of the effects to be considered. Since the half normal line starts at the origin, this produces a more sensitive scale to detect significant outcomes [15], which are immediately detected at a glance. The half normal plot of the experimental values of yield shear stress is shown in Fig. 7. The straight line is built thanks to replicates (triangles) and provides an estimation of the normal distribution of the experimental error, which by definition has a stochastic distribution. The triangles are an expression of the sum of errors [15], which is calculated by Design Expert software [18].

Since both points representing the two variables (pressure and magnetic field) are far from the error line, ANOVA demonstrates that these variables influence the process.

The interaction between the variables is also important, which means that the increase in shear yield stress due to the combined action of pressure and magnetic field is greater than the sum of the two effects taken separately.

The experimental test points have a little dispersion, as demonstrated in Fig. 8, provided by the Design Expert software, where the I-beams bars represent the standard deviation. 


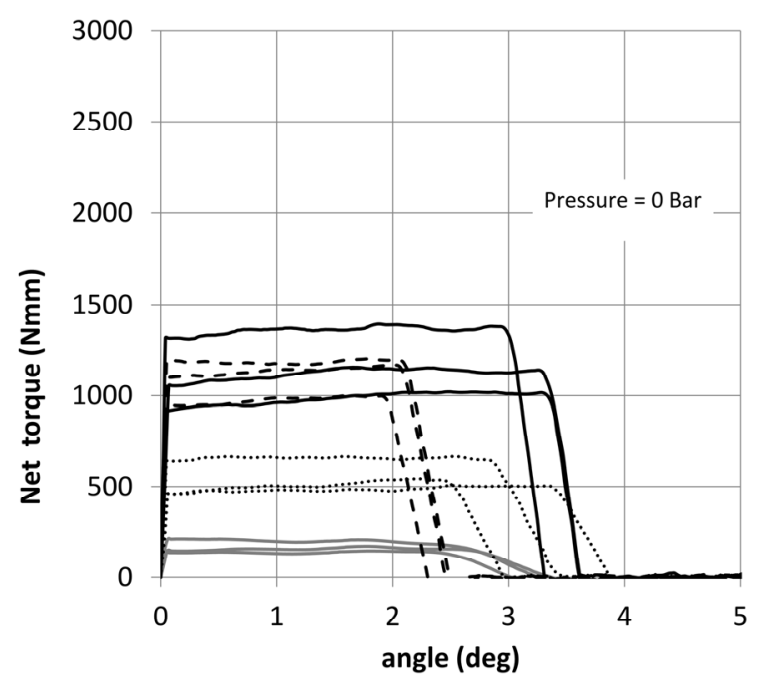

(a)

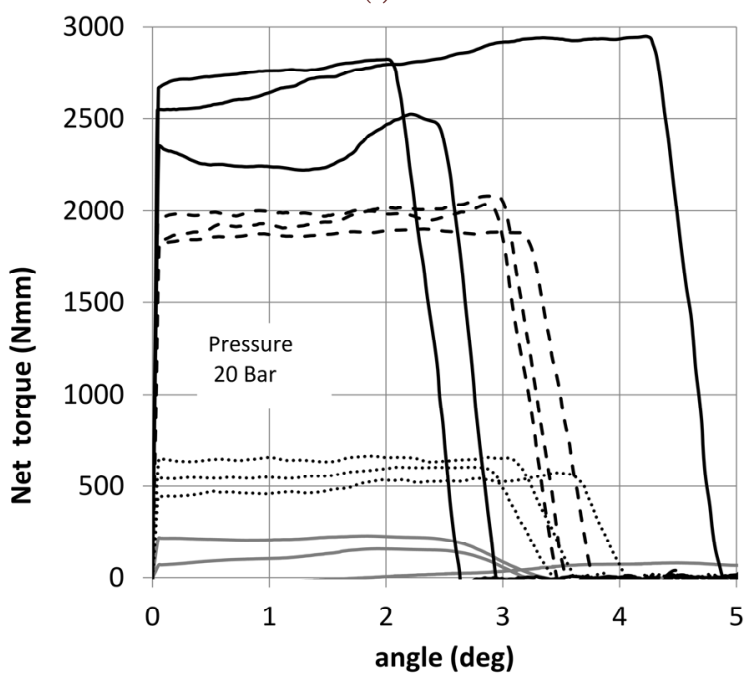

(c)

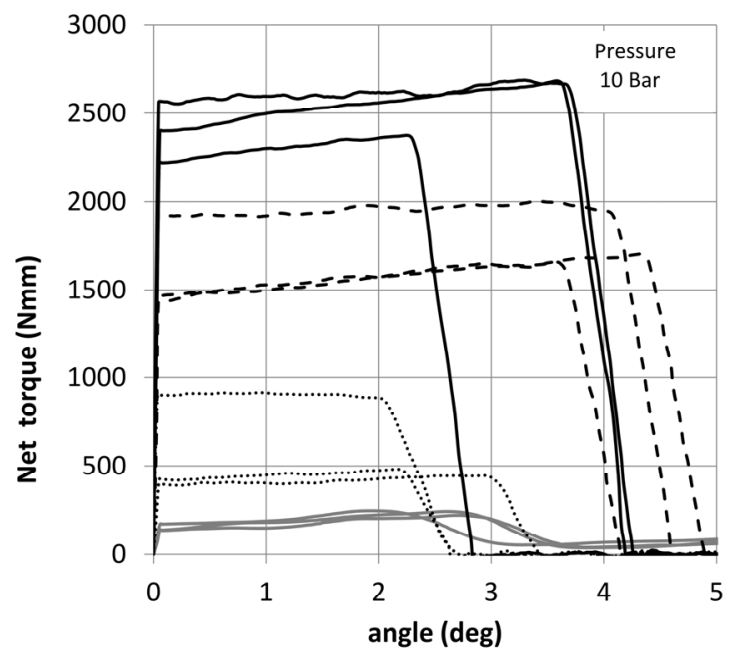

(b)

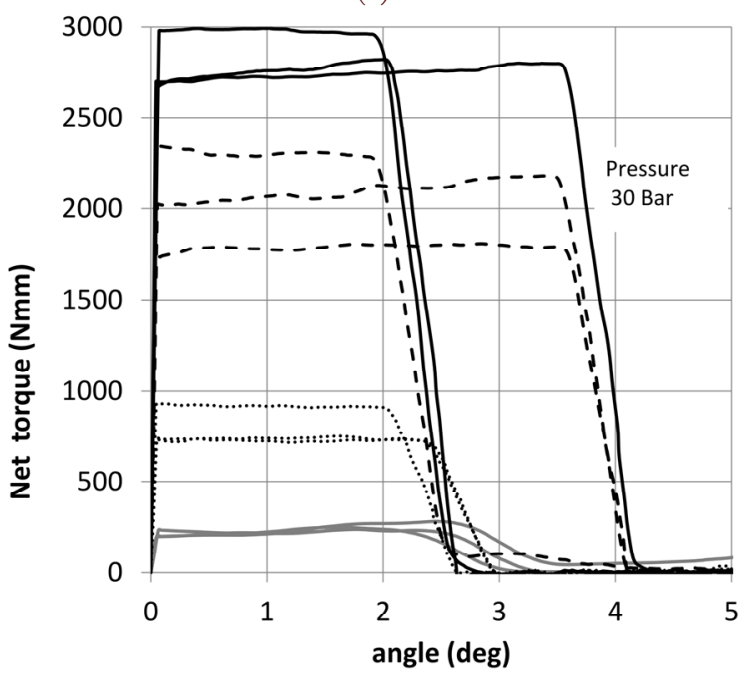

(d)

Figure 6: Experimental torque-angle curves for 50mT (grey solid lines), 100mT (dotted lines), 200mT (dashed line) and 300mT (solid black line). Pressures of 0,10,20 and 30 Bars, reported in (a), (b), (c) and (d), respectively.

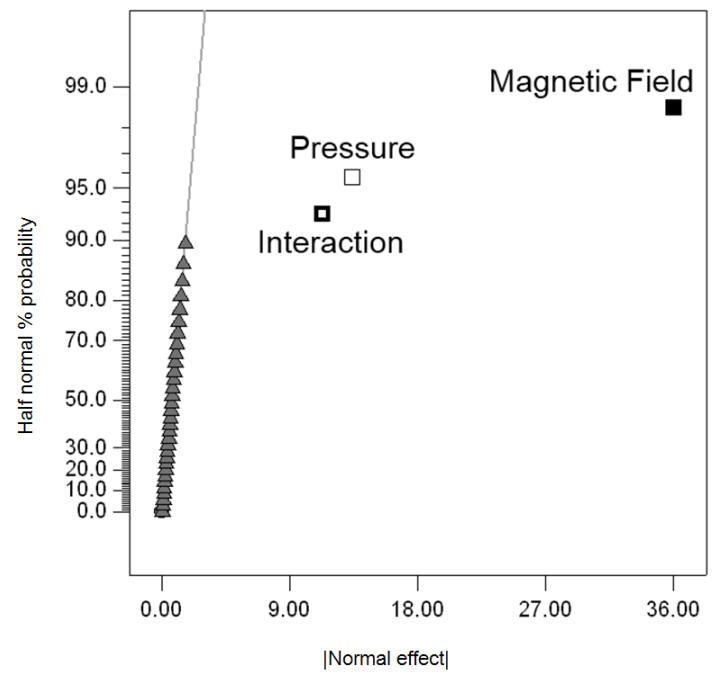

Figure 7: Half normal plot of the yield shear stress: effect of the magnetic field (black square), pressure (white square) and their interaction (hollow black square). 
It is interesting to compare the response surface with the producer experimental curves [2] in order to assess the performances of the MR fluid. The value at no pressure and $50 \mathrm{mT}$ are in good agreement with the producer yield stress, while as the pressure goes up to 30 Bar the yield shear stress has an increment ranging from a $40 \%$ at low magnetic fields up to $200 \%$ for the highest values of magnetic fields. The experimental tests on the whole confirm the findings of [6], [7] and [17] and provide useful information to exploit MR fluids at the maximum of their capabilities.

Exploiting the squeeze strengthening effect could be quite useful and simple in devices like the by-pass damper or the single rod damper, where a pneumatic accumulator is already present in the system. In this case the accumulator pressure could be controlled to enhance MR device performances. In other systems, like the clutches, the applications seems less useful mainly for sealing issues, but still applicable if better performances are needed with tight dimensional constraints.

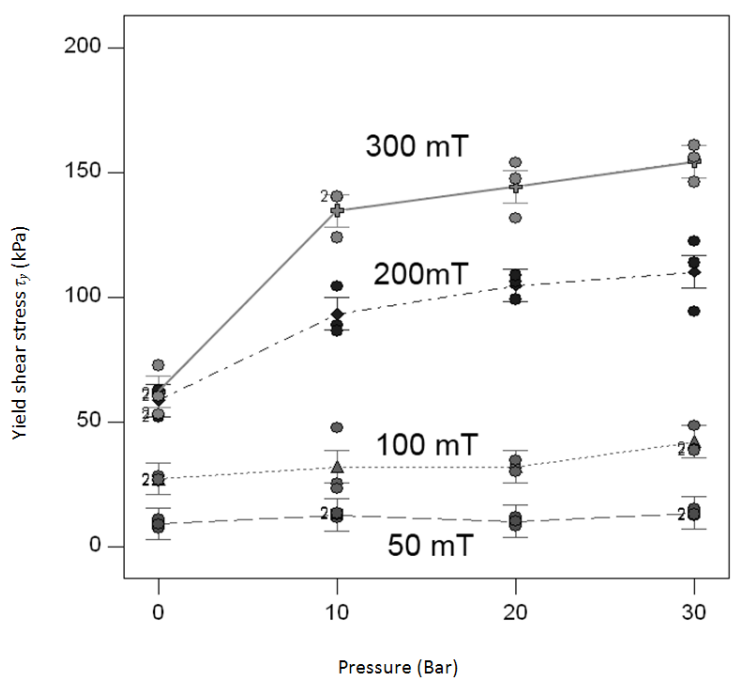

Figure 8: Error bars and experimental yield shear stress for the considered pressure (along the x-axis) and magnetic field levels, 50m T (dashed line), $100 \mathrm{mT}$ (dotted line), $200 \mathrm{~m}$ T (dot-dashed line) and $300 \mathrm{mT}$ (solid line).

\section{CONCLUSION}

7 he paper explored the behaviour of magnetorheological fluids in shear mode under the combined action of a magnetic field and internal pressure. The experimental apparatus was designed and developed to characterize the MR fluids under the rotary action typical of MR brakes and clutches. The experimental tests showed the influence on the shear yield stress both of the magnetic field and of the applied pressure. In addition analysis of variance revealed a positive interaction between the two factors considered in the experimental plan. The shear stress values under pressure were found to be more than two times the values reached with no pressure. The experimental test procedure ensure to eliminates the influence of the sealing and to focus only on the MR effect augmented by the pressure. This MR fluid peculiarity can be exploited to modify existent MR based devices with little changes in system architecture, like in the systems with pneumatic accumulator, but strong improvement in terms of performances.

\section{REFERENCES}

[1] M. R. Jolly, J.W. Bender, J. D. Carlson, In: SPIE $5^{\text {th }}$ Int. Symposium on Smart Structures and Materials, San Diego, CA (1998).

[2] Lord Corporation, Cary, NC http://www.lordfulfillment.com/upload/DS7012.pdf.

[3] J. M. Ginder, L. C. Davis, Appl. Phys. Lett., 65 (1994) 3410.

[4] Lord Corporation, Cary, NC, http://edge.rit.edu/content/P07307/public/LORD\%20MR\%20Fluid.

[5] Lord Corporation, Cary, NC ftp://ftp.elet.polimi.it/users/Luigi.Piroddi/MRD/LORD/MRF132LD.pdf.

[6] X. Tang, X. Zhang, R. Tao, Y. Rong, J. Appl. Phys., 87 (2000) 2634.

[7] X. Zhang, X. L. Gong, P. Q.Zhang, J. Appl. Phys., 96 (2004) 2359.

[8] D. C. Montgomery, Design and analysis of experiments, Wiley, New York (1997). 
[9] E. C. Bingham, Fluidity and Plasticity, McGraw-Hill, New York (1922).

[10] Polypac Ring T.E.F. E/GR http://tinyurl.com/68hy5nu.

[11] Keller-Druck, http:/ / tinyurl.com/3u7p6or.

[12] M. Zubieta, S. Eceolaza, M. J. Elejabarrieta, M. M. Bou-Ali, Smart Mater. Struct., 18 (2009) 095019.

[13] P. Forte, M. Paternò, E. Rustighi, Int. J. Rotating Mach, 10(3) (2004) 175.

[14] FEMM 4.0 - Finite Element Method Magnetics - http://www.femm.info/wiki/HomePage.

[15] M. J. Anderson, P. J. Whitcomb, DOE Simplified: Practical Tools for Effective Experimentation, Productivity Press (2007) .

[16] R. F. Ierardi, A. Bombard, Journal of Physics: Conference Series,149 (2009) 012037.

[17] A. Spaggiari, E. Dragoni, J. Fluids Engineering, 134 (2012) 0091103-1.

[18] Stat-Ease, Design-Expert 8 http:/ / tinyurl.com/yers2w6.

[19] Matlab 7.10, Mathwoks, http://tinyurl.com/jof3k 